\title{
Disproportionate Intrauterine Growth Intervention Trial At Term: DIGITAT
}

\author{
Kim E Boers*1, Denise Bijlenga², Ben WJ Mol3 ${ }^{3}$, Saskia LeCessie ${ }^{4}$, \\ Erwin Birnie ${ }^{5}$, Marielle G van Pampus ${ }^{6}$, Rob H Stigter ${ }^{7}$, \\ Kitty WM Bloemenkamp ${ }^{1}$, Claudia A van Meir ${ }^{8}$, Joris AM van der Post ${ }^{9}$, \\ Dick J Bekedam ${ }^{10}$, Lucy SM Ribbert ${ }^{11}$, Addie P Drogtrop ${ }^{12}$, Paulien CM van \\ der Salm ${ }^{13}$, Anjoke JM Huisjes ${ }^{14}$, Christine Willekes ${ }^{15}$, Frans JME Roumen ${ }^{16}$, \\ Hubertina CJ Scheepers ${ }^{17}$, Karin de Boer ${ }^{18}$, Johannes J Duvekot ${ }^{19}$, \\ Jim G Thornton ${ }^{20}$ and Sicco A Scherjon ${ }^{1}$
}

Address: ${ }^{1}$ Department of Obstetrics and Gynaecology, Leiden University Medical Center, The Netherlands, ${ }^{2}$ Department of Social Medicine, Academic Medical Center Amsterdam, The Netherlands, ${ }^{3}$ Department of Obstetrics and Gynaecology, Máxima Medical Center Veldhoven, The Netherlands, ${ }^{4}$ Department of Medical Statistics and Bio-informatics, Leiden University Medical Center, Tthe Netherlands, ${ }^{5}$ Department of Public Health Economy, Erasmus Medical Center Rotterdam, The Netherlands, ${ }^{6}$ Department of Obstetrics and Gynaecology, University Medical Center Groningen, The Netherlands, ${ }^{7}$ Department of Obstetrics and Gynaecology, Deventer Hospital, The Netherlands, ${ }^{8}$ Department of Obstetrics and Gynaecology, Groene Hart Hospital Gouda, The Netherlands, ${ }^{9}$ Department of Obstetrics and Gynaecology, Academic Medical Center Amsterdam, The Netherlands, ${ }^{10}$ Department of Obstetrics and Gynaecology, OLVG Amsterdam, The Netherlands, ${ }^{1}$ Department of Obstetrics and Gynaecology, St. Antonius Hospital Nieuwegein, The Netherlands, ${ }^{12}$ Department of Obstetrics and Gynaecology, TweeSteden Hospital Tilburg, The Netherlands, ${ }^{13}$ Department of Obstetrics and Gynaecology, Meander Medical Center Amersfoort, The Netherlands, ${ }^{14}$ Department of Obstetrics and Gynaecology, Gelre Hospital Apeldoorn, The Netherlands, ${ }^{15}$ Department of Obstetrics and Gynaecology, University Hospital Maastricht, The Netherlands, ${ }^{16}$ Department of Obstetrics and Gynaecology, Atrium Medical Center Heerlen, The Netherlands, ${ }^{17}$ University Medical Center St. Radboud Nijmegen, The Netherlands, ${ }^{18}$ Rijnstate Hospital Arnhem, The Netherlands, ${ }^{19}$ Department of Obstetrics and Gynaecology, Erasmus Medical Center Rotterdam, The Netherlands and ${ }^{20}$ Department of Obstetrics and Gynaecology and Child Health, University of Nottingham, Nottingham City Hospital, UK

Email: Kim E Boers* - k.e.boers@lumc.nl; Denise Bijlenga - D.Bijlenga@amc.uva.nl; Ben WJ Mol - b.mol1@chello.nl; Saskia LeCessie - S.le_Cessie@lumc.nl; Erwin Birnie - e.birnie@amc.uva.nl; Marielle G van Pampus - m.g.van.pampus@og.umcg.nl; Rob H Stigter - rstigter@planet.nl; KittyWM Bloemenkamp - k.w.m.bloemenkamp@lumc.nl; Claudia A van Meir - Claudia.van.Meir@ghz.nl; Joris AM van der Post - j.a.vanderpost@amc.uva.nl; Dick J Bekedam - dbekedam@xs4all.nl; Lucy SM Ribbert - l.ribbert@antonius.net; Addie P Drogtrop - adrogtrop@tsz.nl; Paulien CM van der Salm - P.Salm@meandermc.nl; Anjoke JM Huisjes - a.huisjes@gelre.nl; Christine Willekes - cwi@sgyn.azm.nl; Frans JME Roumen - f.roumen@atriummc.nl; Hubertina CJ Scheepers - hcj.scheepers@tiscali.nl; Karin de Boer - karinber@planet.nl; Johannes J Duvekot - j.duvekot8@chello.nl; Jim G Thornton - Jim.Thornton@nottingham.ac.uk;

Sicco A Scherjon - S.Scherjon@lumc.nl

* Corresponding author

Published: 10 July 2007

BMC Pregnancy and Childbirth 2007, 7:12 doi:10.1186/1471-2393-7-12
Received: 13 June 2007

Accepted: 10 July 2007

This article is available from: http://www.biomedcentral.com/I47I-2393/7/12

(c) 2007 Boers et al; licensee BioMed Central Ltd.

This is an Open Access article distributed under the terms of the Creative Commons Attribution License (http://creativecommons.org/licenses/by/2.0), which permits unrestricted use, distribution, and reproduction in any medium, provided the original work is properly cited.

\begin{abstract}
Background: Around $80 \%$ of intrauterine growth restricted (IUGR) infants are born at term. They have an increase in perinatal mortality and morbidity including behavioral problems, minor developmental delay and spastic cerebral palsy. Management is controversial, in particular the decision whether to induce labour or await spontaneous delivery with strict fetal and maternal surveillance. We propose a randomised trial to compare effectiveness, costs and maternal quality
\end{abstract}


of life for induction of labour versus expectant management in women with a suspected IUGR fetus at term.

Methods/design: The proposed trial is a multi-centre randomised study in pregnant women who are suspected on clinical grounds of having an IUGR child at a gestational age between $36+0$ and $4 I+0$ weeks. After informed consent women will be randomly allocated to either induction of labour or expectant management with maternal and fetal monitoring. Randomisation will be webbased. The primary outcome measure will be a composite neonatal morbidity and mortality. Secondary outcomes will be severe maternal morbidity, maternal quality of life and costs. Moreover, we aim to assess neurodevelopmental and neurobehavioral outcome at two years as assessed by a postal enquiry (Child Behavioral Check List-CBCL and Ages and Stages Questionnaire-ASQ). Analysis will be by intention to treat. Quality of life analysis and a preference study will also be performed in the same study population. Health technology assessment with an economic analysis is part of this so called Digitat trial (Disproportionate Intrauterine Growth Intervention Trial At Term). The study aims to include 325 patients per arm.

Discussion: This trial will provide evidence for which strategy is superior in terms of neonatal and maternal morbidity and mortality, costs and maternal quality of life aspects. This will be the first randomised trial for IUGR at term.

Trial registration: Dutch Trial Register and ISRCTN-Register: ISRCTNI03632I7.

\section{Background}

Around $80 \%$ of intrauterine growth restricted (IUGR) infants are born at term [1]. When pregnancy is complicated by IUGR, there is, whether term or preterm, a clear association with an increase in neonatal mortality and neonatal morbidity (short and long term) [2-4]. The long term morbidity ranges from behavioral problems and minor developmental delay to spastic cerebral palsy [510]. However, not all studies, especially after excluding congenital anomalies, confirm these findings [11]. Besides fetal asphyxia, meconium aspiration, fetal heart rate abnormalities and low Apgar score, also more admittances to and longer stays at neonatal intensive care units are reported. This might partly be related to a higher prevalence of hypoglycaemia, neonatal sepsis, hypothermia and haematological problems as thrombocytopenia and polycythemia in these neonates [12-14].

When a fetus is small for gestational age (SGA), defined on the basis of a birth weight below the $10^{\text {th }}$ centile, there is the concern that the fetus might be afflicted by IUGR [15]. As SGA is defined on the basis of an arbitrary chosen cutoff birth weight centile, not all infants falling below the $10^{\text {th }}$ centile are abnormally small because of growth restriction. Many neonates with a birth weight below the $10^{\text {th }}$ centile are representing the normal spectrum of fetal growth [11]. Variation in birth weight is related to many factors as maternal height, weight, parity and fetal gender, but also ethnicity [16]. For that reason optimal growth for any fetus should be related to the fetus' own individual optimal growth curve [17-19]. Intrauterine growth restriction has to be defined on further knowledge such as Doppler abnormalities as seen in placental perfusion, eventually in combination with abnormalities in cerebral perfusion [20,21] and possibly also by neonatal measurements as the Ponderal Index [22,23].

A reduction of fetal growth is exponentially associated with a higher perinatal mortality [24] and morbidity $[25,26]$. Doppler umbilical artery studies have shown that absence of end diastolic velocities, indicative of IUGR based on severe placental insufficiency is associated with a higher rate of caesarean deliveries and an increased incidence of perinatal and neonatal mortality [27-30]. However, a normal umbilical artery Doppler study at term gestation might be falsely reassuring, while a normal cerebral artery study might identify the fetus not likely having a major adverse outcome [31].

Most of the growth restricted children experience an accelerated growth, especially of the head circumference, during the first 6 months after birth [32]. However, this upward centile crossing or 'catch up growth' is not complete, even at the age of seven years [33]. Moreover head circumference seems to correlate with cognitive outcome [34].

Long-term neurological and cognitive development of the IUGR infant at term have been studied extensively. The Ponderal Index among IUGR infants, but also among infants with a normal birth weight, is an independent predictor of neonatal morbidity: the lower the Ponderal Index the higher morbidity [25]. Learning difficulties, defects in speech and mild neurological deficits and behavioral problems have been reported to occur more in term neonates born SGA $[35,36]$. At school ages (7-8 
years) temperamental differences and differences in play behavior are apparent [37], most probably contributing to increased rate of school failure found in IUGR infants.

Long-term morbidity might be resulting from subtle nutritional insults to the brain in utero. Although the brain growth spurt, being the most vulnerable period of the human brain, spans a broad period between mid pregnancy and 6 months of postnatal age [38,39], it is shown that growth failure occurring around term shows a strong association with cognitive disturbances as a poorer mental and psychomotor development at two years of age [40]. However, not all studies, even at preschool age show this trend of increased problems in growth restricted infants $[41,42]$. Besides neurodevelopmental consequences it is now also clear that children who were undernourished during pregnancy (e.g. born with a birth weight more than 2 SD below the mean birth weight) and especially in combination having had a compensatory growth trajectory during childhood have an increased risk in later life for diabetes, hypertension and cardiovascular diseases [43].

Given the data from studies concerning the effect of under-nutrition on the brain and the effects on long-term cognitive and behavioral outcome, evaluation of the possible clinical benefit of early induction of delivery, preempting a detrimental effect of chronic under nutrition on the fetal brain intervention, is important. By such an intervention it might be possible to start earlier with a more optimal feeding, compensating for the poor intrauterine environment. Induction of labor is very often common practice in cases of suspected IUGR $[44,45]$. In the Netherlands at 33 up to 36 weeks of gestation, $63 \%$ of IUGR pregnancies were induced, whereas from 37 weeks onwards this percentage is $23 \%$; more than double the percentage in non-IUGR pregnancies. In a Dutch obstetric cohort of 14.294 primigravid women with IUGR pregnancies, $29 \%$ of these pregnancies were induced [46]. In these pregnancies complicated by IUGR, induction of labour was associated with an increased risk of instrumental deliveries and emergency caesarean section, but no difference in neonatal outcome immediately after birth was found.

At present, there is no uniformity on the management of women with IUGR at term. Although there is no doubt that the intra-uterine growth retardated fetus should be considered as high risk, and should be monitored, there is no consensus on which diagnostic methods to evaluate fetal condition and subsequent intervention is best. It is unclear whether in this situation either induction of labour or expectant management is beneficial for the mother and her baby, since evidence on the subject is lacking.
For preterm pregnancies complicated by intra-uterine growth retardation, an international randomised clinical trial recently showed that expectant management had little benefit over early delivery with respect to short term neonatal outcome [47]. However, results of this trial cannot be extrapolated to the situation at term.

The lack of consensus on the subject in the Netherlands is demonstrated by the fact that in 2002 in women with a SGA child, labour was induced in $32 \%$ of these women, whereas labour started spontaneously in $56 \%$ of these women, the remaining $11 \%$ had an elective caesarean section. These data are based on actual birth weight, and the clinical situation is even more complicated by the fact that the antenatal diagnosis of a SGA child is often difficult to make and easily missed in clinical practice.

In view of this clinical dilemma, we propose a randomised clinical trial in which induction of labour is compared with expectant monitoring in women with a suspected IUGR child at term. We will compare maternal outcome, neonatal outcome and maternal quality of life, as well as costs. Moreover, we will collect, in both randomisation arms, data of the diagnostic tests used in fetal surveillance, i.e. fetal heart rate pattern, sonographic measurement of the amniotic fluid index and Doppler measurement of the umbilical artery and the fetal medial cerebral artery in women.

\section{Methods/design}

Aims

The aim of this study is to investigate whether induction of labour or expectant management is the best strategy in terms of neonatal and maternal morbidity and mortality, costs and maternal quality of life aspect in pregnancies complicated by IUGR from 36 weeks gestational weeks onwards.

\section{Study design and setting}

We will perform a randomised controlled multi centre study.

This trial is embedded in the Dutch Obstetric Consortium, a collaboration of obstetric hospitals in the Netherlands. Approximately 40 hospitals, including all 10 university hospitals, teaching hospitals and district hospitals will participate in this trial.

\section{Participants/eligibility criteria}

All women with a singleton pregnancy, with a child in cephalic presentation, with suspicion of IUGR (Fetal Abdominal Circumference $<10^{\text {th }}$ centile, Estimated Fetal Weight $<10^{\text {th }}$ percentile as defined by local protocols), or decreased relative growth though still $>10^{\text {th }}$ centile, e.a. from $70^{\text {th }}$ centile to $40^{\text {th }}$ centile) are eligible. Gestational 
age should be between $36+0$ weeks and $41+0$ weeks. Women with a history of caesarean section, serious congenital defects, ruptured membranes, renal diseases, diabetes mellitus, or positive HIV serology will be excluded.

\section{Procedures, recruitment, randomisation and collection of} baseline data

All women with a singleton pregnancy who present at one of the participating clinics will be referred to an obstetrician or a specifically appointed research nurse/midwife for counselling. Eligible women receive participant information. After written consent, they are randomised by means of a web-based application. Stratification will be applied for previous vaginal birth (nullipara versus multipara) and for centre. Randomisation will be in a 1:1 ratio for induction of labour or expectant management.

Patients that withhold consent for randomisation are asked permission for data collection on pregnancy outcome. Participation to the quality of life study and longterm follow up (Child Behavioural Check Lists-CBCL and Ages and Stages Questionnaire-ASQ) is asked separately.

Baseline demographic, past obstetric and medical histories will be recorded for all women. Cervical length will be measured at the time of randomisation. The quality of life questionnaires are filled out before randomization, after randomization, 6 weeks postpartum and 6 months postpartum. The questionnaires contain the Hospital Anxiety and Depression Scale (HADS), EuroQoL 5D3L, Short Form (SF-36), Symptom Check List (SCL-90), and questions on background characteristics, intervention preparedness, risk perception and experience with the current pregnancy.

\section{Intervention}

When randomised to the induction arm, induction of labor must start within 48 hours after randomisation. Induction of labor can be proceded according to local protocol (among other things cervical ripening with prostaglandin-gel or tablets or with amniotomy, with or without the use of oxytocin). When allocated to the expectant management group patients will not be induced unless the fetal or maternal condition deteriorates and this is for the attending obstetrician a reason for induction. The patients will be observed, e.g. with fetal and maternal monitoring according to local practice, until labour starts spontaneously. However, monitoring must at least include measurement of the umbilical artery Doppler waveform, fetal heart rate tracing, blood pressure and urine analysis for albuminuria weekly. Doppler studies of the medial cerebral artery are optional. Reasons for interventions and time interval between randomisation and labour will be collected.

\section{Follow up of women and infants}

All details of delivery, maternal and fetal assessments and admittance during pregnancy are recorded in the case record form that is accessible at the website. In case of admittance of the child to the neonatal intensive care unit, details of this admittance are also recorded.

Long-term follow up of children will be done by recording growth after birth as measured at the local infant follow up clinics.

\section{Outcome measures}

The primary outcome measure will be a bad composite neonatal outcome. Adverse neonatal outcome will be defined as death before hospital discharge, a 5-minute Apgar score $<7$, an umbilical artery $\mathrm{pH}<7.05$ or admission to the neonatal intensive care. Secondary outcome measures are mode of delivery and time until delivery, length of admittance at the neonatal intensive care, maternal morbidity, hospitalisation of the mother for fetal and maternal surveillance, quality of life, and costs. In the present proposal, no funding is asked for long term follow-up of the child, yet. However, if additional funding can be obtained children's behavioural-, and neuro development will assessed by administering with a postal enquiry the Child Behaviour Checklist-CBCL and Ages and Stages Questionnaire- ASQ by their parents after 2 years.

\section{Statistical issues}

Sample size calculations

The study is designed as an equivalence study, whereby both treatments will have the same incidence of the primary outcome measure of combined bad neonatal outcome. This incidence is assumed to be 6\% [46]. The null hypothesis is that both treatments will not be equivalent. To detect equivalence with a power of $80 \%$ a sample size in both groups of 325 will be needed (PASS SOFTWARE). The margin of equivalence, given in terms of the difference, extends from $-5.5 \%$ to $+5.5 \%$. The actual difference is $0 \%$. The calculations assume that two, one-sided $\mathrm{Z}$ tests are used. The significance level of the test is 0.05 .

\section{Data analysis}

Data will initially be analysed according to the intention to treat method. The main outcome variable, 'bad neonatal outcome', will be assessed by calculating rates in the two groups, relative risks and $95 \%$ confidence intervals as well as numbers needed to treat.

Time to delivery will be evaluated by Kaplan-Meier estimates, with account for differing durations of gestation at entry, and will be tested with the log rank test. The other secondary outcome measures will be approached simi- 
larly to the primary outcome measure. The analysis will be stratified for parity and centre.

\section{Non response and inclusion bias}

As non-response for follow up is overrepresented in certain outcome-related risk categories such as in non-native mothers, mothers with lower educational level and in mothers with boys, statistical methods that use imputation of missing data have to be applied [48]. To prevent inclusion bias all patients who were asked but decline randomisation, will be asked for permission to collect data on pregnancy outcome and further follow up according to the same schedule as the randomised patients.

\section{Economic evaluation}

The aim of the economic evaluation is to compare optimality, in terms of costs and health effects, of both strategies. As the clinical study is based on equivalence design we hypothesize that there will be no relevant difference between maternal and neonatal outcome in the two strategies. The economic evaluation will be in the form of a cost-effectiveness analysis (CEA), in which the optimal strategy is defined as the strategy with the largest health gain at the smallest costs.

\section{Ethical considerations}

This study has been approved by the ethics committee of the Leiden University Medical Centre (Ref. No. P04.210).

\section{Discussion}

There is uncertainty about the management of IUGR at term, whether to leave the child in utero until spontaneous labour starts, or to prevent undernutrition by prolonged pregnancy in a poor intra-uterine environment by inducing labour. This latter treatment modality will most probably be at the cost of an increase in instrumental deliveries [46]. As optimal management of a pregnancy at term suspected to be complicated by IUGR remains unclear, it is a challenge to develop criteria for inducing delivery. An increase in fetal surveillance in these pregnancies (with normal umbilical artery studies) is thought to be associated with more inductions of labour and a shortening of gestational age [49]. Neonatal morbidity (and mortality) is low in term SGA neonates [3], nevertheless these neonates cannot be considered just "healthy small babies".

Although our primary aim is to study pregnancies complicated by IUGR, the inclusion criteria are obviously based on a suspicion of a SGA child, as we include women with a fetus with a Fetal Abdominal Circumference $<10^{\text {th }}$ centile or an Estimated Fetal Weight $<10^{\text {th }}$ centile. By patient's characteristics, such as ethnicity, maternal and paternal length as well as tests results as the amount of amniotic fluid or the Doppler of the arteria umblicalis, we will be able to evaluate which pregnancies are at risk for a poor neonatal outcome.

In summary, at the present, there is controversy as to which strategy is the best when IUGR at term is suspected. Whether to induce labour or to await spontaneous labour under strict fetal and maternal monitoring remains debatable because of a lack of evidence. Patients' management partly depends on the attending doctor and on local protocols. To resolve these issues, we will compare both strategies in the multi centre randomised trial - DIGITAT. In a pilot study carried out in one of the participating hospitals, we examined the feasibility of the DIGITAT-trial. Preliminary data from this small pilot show that the interval between randomisation and labour was 2 weeks shorter and birth-weight was 100 grams less in the pregnancies that were directly terminated by induction [50]. The results of the present DIGITAT-trial are expected in 2009.

\section{Abbreviations}

IUGR - Intrauterine growth retardation

SGA - Small for gestational age

CBCL - Child Behavioural Check List

ASQ - Ages and Stages Questionnaire

NICU - Neonatal Intensive Care Unit

\section{Competing interests}

The author(s) declare that they have no competing interests.

\section{Authors' contributions}

SS, JvdP, BWM and JGT were involved in conception and design of the study. KEB and SS drafted the manuscript. All authors have read and given final approval of the final manuscript.

\section{Acknowledgements}

This study is funded by ZonMW grant 945-04-558.

\section{References}

I. Clausson B, Cnattingius S, Axelsson O: Preterm and term births of small for gestation age infants: population-based study of risk factors among nulliparous women. Br J Obstet Gynecol I 998, 105:1011-17.

2. Balcazar H, Keefer L, Chard T: Use of anthropometric indicators and maternal risk factors to evaluate intrauterine growth retardation in infants weighing more than 2500 grams at birth. Early Hum Dev 1994, 36: |47-55.

3. Chang TC, Robson SC, Spencer JAD, Gallivan S: Prediction of perinatal mortality at term in small fetuses: comparison of foetal growth and Doppler ultrasound. Br J Obstet Gynaecol I994, iol:422-7.

4. Boulet SL, Alexander GR, Salihu HM, Kirby RS, Carlo WA: Fetal growth risk curves: defining levels of fetal growth restriction by neonatal death risk. Am J Obstet Gynecol 2006, I 95: I57|-7.

5. Dijxhoorn MJ, Visser GHA, Touwen BCL, Huisjes HJ: Apgar score, meconium and acidaemia at birth in smallforgestational age 
infants born at term, and their relation to neonatal neurological morbidity. $\mathrm{Br}$ J Obstet Gynaecol 1987, 94:873-9.

6. Soothill PW, Ajayi RA, Campbell S, Nicolaides KH: Prediction of morbidity in small and normally grown fetuses by fetal heart rate variability, biophysical profile score and umbilical artery Doppler studies. Br J Obstet Gynaecol 1993, 100:742-52.

7. Low JA, Galbraith RS, Muir D, Killen H, Karchmar J, Campbell D: Intrauterine growth retardation: a preliminary report of long-term morbidity. Am / Obstet Gynecol 1978, I 30:534-45.

8. Harvey D, Prince J, Bunton J, Parkinson C, Campbell S: Abilities of children who were small-for-gestational-age babies. Pediatrics 1982, 69:296-300.

9. Paz I, Gale R, Laor A, Danon YL, Stevenson DK, Seidman DS: The cognitive outcome of fullterm small for gestational age infants at late adolescence. Obstet Gynecol 1995, 85:452-6.

10. Blair E, Stanley F: Intrauterine growth and spastic cerebral palsy. Am J Obstet Gynecol 1990, 162:229-37.

II. Chard T, Yoong A, Macintosh M: The myth of foetal growth retardation at term. Br J Obstet Gynaecol 1993, 100:1076-81.

I2. Battaglia FC: Intrauterine growth retardation. Am J Obstet Gynecol 1970, 106:1 103-14.

13. Doctor BA, O'Riordan MA, Kirchner HL, Shah D, Hack M: Perinatal correlates and neonatal outcomes of small for gestational age infants born at term gestation. Am J Obstet Gynecol 200I, I 85:652-9.

14. Minior VK, Divon MY: Fetal growth restriction at term: myth or reality? Obstet Gynecol 1998, 92:57-60.

15. Deter RL, Harrist RB, Hill RM: Neonatal growth assessment score: a new approach to the detection of intrauterine growth retardation in the newborn. Am J Obstet Gynecol 1990, 162:1030-1036.

16. Drooger JC, Troe JWM, Borsboom GJJM, Hofman A, Mackenbach JP, Moll HA: Ethnic differences in prenatal growth and the association with maternal and fetal characteristics. Ultrasound Obstet Gynecol 2005, 26: I I5-22.

17. Deter RL: Individualized growth assessment: evaluation of growth using each fetus as its own control. Semin Perinatol 2004, 28:23-32.

18. Gardosi J, Chang A, Kalyan B, Sahota D, Symonds EM: Customised antenatal growth charts. Lancet 1992, 339:283-7.

19. Mongelli M, Gardosi ]: Reduction of false-positive diagnosis of fetal growth restriction by application of customized fetal growth standards. Obstet Gynecol 1996, 88:844-848.

20. Larciprete G, Valensise H, Di Pierro G, Vasapollo B, Casalino B Arduini $D$ : Intrauterine growth restriction and fetal body composition. Ultrasound Obstet Gynecol 2005, 26:258-62.

21. Bahado-Singh RO, Kovanci E, Jeffres A, Oz U, Deren O, Copel J: The Doppler cerebroplacental ratio and perinatal outcome in intrauterine growth restriction. $\mathrm{Am}$ J Obstet Gynecol 1999, 180:750-6.

22. Walther FJ, Ramaekers LH: The ponderal index as a measure of the nutritional status at birth and its relation to some aspects of neonatal morbidity. J Perinat Med 1982, 10:42-7.

23. Ott W]: Small for gestational age fetus and neonatal outcome: reevaluation of the relationship. Am J Perinat 1995, I 2:396-400.

24. Myers SA, Ferguson R: A population study of the relationship between fetal death and altered fetal growth. Obstet Gynecol 1989, 74:325.

25. Villar J, deOnis M, Kestler E, Bolanos F, Cerezo R, Bernedes H: The differential neonatal morbidity of the intrauterine growth retardation syndrome. Am J Obstet Gynecol 1990, 163:151-157.

26. Balcazar $\mathrm{H}$, Haas J: Classification schemes of small-for-gestational age and type of intrauterine growth retardation and its implications to early neonatal mortality. Early Hum Developm 1990, 24:219-230.

27. Scherjon SA, Smolders-DeHaas H, Kok JH, Zondervan HA: The "brain-sparing" effect: Antenatal cerebral Doppler findings in relation to neurologic outcome in very preterm infants. Am Obstet Gynecol 1993, 169:169-175.

28. Valcamonico A, Danti L, Frusca T, et al.: Absentend-diastolic velocity in the umbilical artery: risk of neonatal morbidity and brain damage. Am / Obstet Gynecol 1994, I 70:796-80I.

29. Steiner H, Staudach A, Spitzer D, Schaffer KH, Gregg A, Weiner CP. Growth deficient fetuses with absent or reversed umbilical artery end-diastolic flow are metabolically compromised. Early Hum Dev 1995, 4I: I-9.

30. Li H, Gudmundsson S, Olofsson P: Prospect for vaginal delivery of growth restricted fetuses with abnormal umbilical artery blood flow. Acta Obstet Gynecol Scand 2003, 82(9):828-33.

31. Fong KW, Ohlsson A, Hannah ME, Grisaru S, Kingdom J, Cohen H: Prediction of perinatal outcome in fetuses suspected to have intrauterine growth restriction: Doppler US study of fetal cerebral, renal, and umbilical arteries. Radiology 1999 , 213:681-9.

32. Watt J: Small-for-gestational age infants: perinatal, physical and social factors in the first year of life. NZ Med J 1989 102:469-472

33. Walther FJ: Growth and development of term disproportionate small-for-gestational age infants at the age of 7 years. Early Hum Develop 1988, I8: I-II.

34. Westwood M, Kramer MS, Munz D, Lovett JM, Watters GV: Growth and development of full-term nonasphyxiated small-for-gestational-age newborns: follow-up through adolescence. Pediatrics 1983, $71(3): 376-82$

35. Low JA, Handley-Derry MH, Burke SO, Peters RD, Pater EA, Killen $\mathrm{HL}$ : Association of intrauterine fetal growth retardation and learning deficits at age 9 to I I years. Am J Obstet Gynecol 1992. 167(6): 1499-505

36. Hill RM, Verniaud W, Deter RL, et al:: The effect of intrauterine malnutrition on the term infant. A 14 year progressive study. Acta Paediatr Scand 1984, 73:482-487.

37. Parkinson CE, Scrivener R, Graves L, Bunton J, Harvey D: Behaviora differences of school-age children who were small-for-dates babies. Develop Med Child Neurol 1986, 28:498-505.

38. Dobbing J, Sands J: Quantitative growth and development of human brain. Arch Dis Child 1973, 48:757-767.

39. Dobbing J, Sands J: Comparative aspects of the brain growth spurt. Early Human Developm 1979, 3:79-83.

40. Skuse D, Pickles A, Wolke D, Reilly S: Postnatal growth and mental development: evidence for a "sensitive period". I Child Psychol Psychiat 1994, 35:521-545.

41. Babson S, Kangas J: Preschool intelligence of undersized term infants. Am I Dis Child 1969, I I 7:553-557.

42. Drillien CM, Thomson A, Burgoyne K: Low-birthweight children at early school-age: a longitudinal study. Develop Med Child Neurol 1980, 22:26-47.

43. Barker DJP, Osmond C, Forsén TJ, Kajantie E, Eriksson JG: Trajectories of Growth among Children Who Have Coronary Events as Adults. NEJM 2005, 353:1802-1809.

44. Xenakis EJ, Piper JM, Conway DL, Langer O: Induction of labor in the nineties: conquering the unfavorable cervix. Obstet Gynecol 1997, 90:235-239.

45. Larsen T, Larsen JF, Petersen S, Greisen G: Detection ofsmall-forgestational-age fetuses by ultrasound screening in a high risk population: a randomized controlled study. Br J Obstet Gynecol 1992, 99(6):469-74

46. Landelijke Neonatale Registratie (Dutch Neonatal Registration). Prismant :2000-2005.

47. GRIT Study Group: A randomised trial of timed delivery for the compromised preterm fetus: short term outcomes and Bayesian interpretation. BJOG 2003, I I O(I):27-32.

48. Hille ET, Elbertse L, Gravenhorst JB, Brand R, Verloove-Vanhorick SP. Dutch POPS-19 Collaborative Study Group. Nonresponse bias in a follow-up study of 19-year-old adolescents born as preterm infants. Pediatrics 2005, I I 6:e662-6.

49. McCowan LM, Harding JE, Roberts AB, Barker SE, Ford C, Stewart AW: A pilot randomized controlled trial of two regimens of fetal surveillance for small-for-gestational-age fetuses with normal results of umbilical artery doppler velocimetry. Am J Obstet Gynecol 2000, 182:81-6.

50. van den Hove MMI, Willekes C, Roumen FJME, Scherjon SA: Intrauterine growth restriction at term: Induction or spontaneous labour?: Disproportionate intrauterine growth intervention trial at term (DIGITAT): A pilot study. Eur J Obstet Gynecol Reprod Biol 2006, I 25:54-8.

\section{Pre-publication history}

The pre-publication history for this paper can be accessed here:

http://www.biomedcentral com/1471-2393/7/12/prepub 